\title{
Patient simulé
}

\author{
En 35 ans de théâtre, j'ai vécu quelques situations inattendues, voire bizarres; mais jouer le malade \\ pour de vrais étudiants, c'était une première.
}

\section{André Cortessis}

Veuillez lire à ce sujet aussi l'article suivant de $\mathrm{R}$ Bonvin.

Pour des raisons de confidentialité, les indications sur les rôles joués ont été modifiées.
Eté 2014. Ciel couvert. Appel d'une amie metteur en scène. On engage des comédiens pour «jouer» les malades lors des examens finaux des étudiants en médecine (ECOS) à Lausanne. Surprise. En 35 ans de théâtre, j'ai vécu quelques situations inattendues, voire bizarres; mais jouer le malade pour de vrais étudiants, ça serait une première. Rendez-vous est pris pour un premier contact avec les responsables de l'Unité de pédagogie au CHUV.

Je dois décrire des symptômes physiques que je n'ai jamais éprouvé...

Après-midi torride, dans un bureau exigu de l'ancien hôpital à la rue du Bugnon. On me donne quelques minutes pour mémoriser une brève anamnèse, pendant qu'une de ces dames revêt une blouse blanche. Je passe en "consultation" avec succès. Questionnaire général, photo, signature d'un contrat de confidentialité.

Après-midi tempétueux, pluie diluvienne, même quartier. Après avoir erré autour et dans un bâtiment au nom ésotérique (Somesthésie!) qui paraît abandonné, je finis par trouver une salle où je suis attendu pour un "screening». Soit une auscultation par un médecin de l'unité pédagogique afin de vérifier que lors des examens, les candidats ne puissent rien déceler d'anormal. Tout va bien, me rassure-t-on, je suis en bonne santé.

Matin ensoleillé et venteux, chez moi. La boîte aux lettres recèle une enveloppe à entête officielle conte-

nant un scénario de trois pages et une convoca-

tion pour deux répétitions, la première dans une semaine. Je dois mémoriser en détail l'heureux passé révolu et le triste présent de Monsieur Y, 70 ans, pensionnaire d'un EMS*.

Matin calme pour une fois, idéal pour un tour en vélo; mais c'est jour de répétition, et je rencontre mes collègues. Car chaque rôle est appris par quatre personnes, qui fonctionneront en alternance le jour de l'examen: deux «au front» et deux en réserve (back-up). Dans une salle de cours du Biopôle IV d'Epalinges, nous visionnons d'abord sur écran la

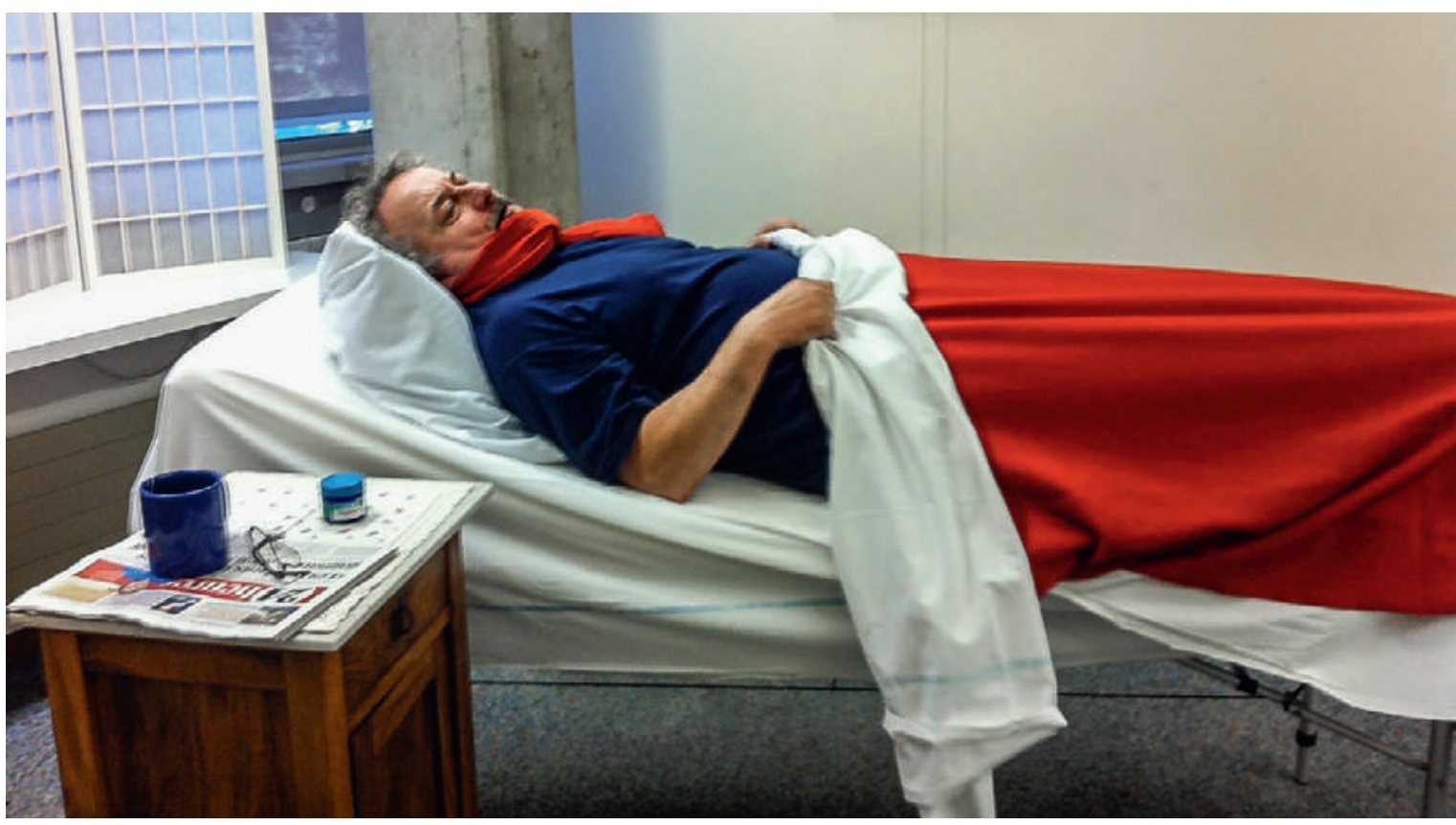

Engagé pour «jouer» les malades lors des examens finaux des étudiants en médecine. 
situation type jouée par deux Bernois (en allemand). Puis notre coach endosse la blouse de candidate, qui a treize minutes pour nous questionner, émettre un diagnostic et proposer un traitement. Deux «phrases magiques» doivent impérativement être prononcées textuellement par le patient au début de l'entretien: Bonjour, depuis quelques temps, je me sens fatigué.Je n'ai plus d'appétit*. Cela n'a l'air de rien, mais c'est plus difficile à placer qu'il n'y paraît... Au bout du premier exercice, un seul des quatre patients simulés a réussi à prononcer les deux phrases... il reste du travail à faire. Règle d'or pour le patient simulé: pas de cadeau (le cadeau, c'est une information donnée spontanément, sans que le candidat ait posé la question idoine).

Jour J, lundi $1^{\mathrm{er}}$ septembre, bise glaciale, Biopôle IV d'Epalinges, 7h3o. L'immense salle du rez-de-chaussée - encore inachevée, béton brut, plastiques de chantier et électricité de fortune - se remplit peu à peu de patients simulés, de tous âges et des deux sexes. J'apprends que nous sommes plus de huitante. Il n'y a pas que des comédiens, et certains sont des habitués de longue date. Café-croissants, on bavarde. Le bâtiment a été savamment aménagé: pas de contact possible entre les candidats et les patients simulés, on circule par des escaliers et à des horaires différents, de façon à ne jamais se rencontrer. On nous appelle. Une petite pièce toute blanche, juste assez grande pour contenir un lit d'examen et un fauteuil pour le "patient», et un petit bureau pour l'expert qui notera le candidat. Affichée sur la porte, une brève description du "cas» à l'attention du candidat, qui la découvre au moment où retentit la sonnerie du compte à rebours. Il frappe, entre, salue et se présente. Après 11 minutes, une sonnerie retentit et une voix sort des haut-parleurs: Plus que 2 minutes. Un peu plus tard, nouvelle sonnerie: Veuillez quitter la salle. Et en route vers le cas suivant. Je préfère être à ma place qu'à la leur...

Sept candidats vont se succéder ainsi pendant la première période. En fait, une majorité de candidates. Le trac est parfois perceptible, la main tendue tantôt moite, tantôt glacée, mais aussi parfois ferme et chaleureuse. Je capte souvent de l'empathie, une main sur l'épaule, des paroles de réconfort pour ce pauvre Monsieur Y. Petit à petit mon inquiétude de néophyte dans ce rôle s'estompe, le médecin expert trouvant ma prestation convaincante.

Mercredi 3 septembre, ciel bleu et bise toujours, même lieu mais 7 h15 cette fois. Pour la troisième journée des examens fédéraux, changement de personnage et de pathologie: me voici dans la peau et sous les pansements de Monsieur Z, 68 ans, fraîchement opéré et angoissé par des complications de plus en plus pénibles*. Cette fois, une sérieuse séance de maquillage s'impose, suivie de la pose (fictive) d'un goutte-à-goutte et de lunettes à oxygène.

Et c'est en blouse d'hôpital que je passe à la phase café-croissant. Ce matin, je commence par une période en réserve. Je suis tendu. Lundi j'ai eu l'impression de bien m'en tirer, le personnage du pensionnaire d'EMS m'a convenu. Mais aujourd'hui c'est différent; je dois décrire des symptômes physiques que je n'ai jamais éprouvé... Et si un candidat venait à échouer par ma faute, induit en erreur par une mauvaise réponse? Un trac d'un nouveau genre me saisit. Sur scène, une faute de texte est la plupart du temps anodine, on se rattrape comme on peut et la plupart

Et si un candidat venait à échouer par ma faute, induit en erreur par une mauvaise réponse?

du temps le public n'y voit que du feu; mais là? Je lis et relis l'anamnèse de Monsieur Z Concentration maximum. $18 \mathrm{~h}$. La vingt-et-unième et dernière candidate de cette folle journée referme la porte de ma chambre. J'ai été palpé et ausculté autant de fois. Une candidate m'a glissé: Vous surveillez ces grains de beauté, Monsieur $\mathrm{Z}$ ? J'espère qu'elle a eu droit à un point supplémentaire.

18h3o, dans le métro. Fatigué mais content, je reconnais une des candidates de ce matin, elle s'assied à mes côtés.

- Cela ne vous a pas gênée d'avoir à examiner des faux malades?

- Pas du tout, au contraire, je me suis sentie moins stressée que si j'avais dû accomplir les mêmes gestes avec de vrais patients.

- Quels sont vos projets?

- Je pars en vacances demain.

- Mais encore?

- Je pense entreprendre une spécialisation en médecine générale.

- Merci de ne pas oublier les patients de la campagne!

Je ressors de ces deux journées intenses impressionné par les qualités de tous ces futurs/res médecins, et prêt à renouveler l'expérience l'an prochain. 